\title{
The proteome of remyelination is different from that of developmental myelination
}

Joana Paes de Faria1, Maria M. Azevedo1, Damaris Bausch-Fluck2, Ana Seixas1, Helena S.

Domingues1, Maria A. Monteiro1, Patrick G.A. Pedrioli2,3,4, Eduarda Lopes1, Rui

Fernandes1, Chao Zhao5, Robin J. M. Franklin5, Bernd Wollscheid2, Joao B. Relvas1,6*, Laura Montani1,7*\#

1Instituto de Biologia Molecular e Celular, i3S Instituto de Investigação e Inovação em Saúde, Universidade do Porto, 4200-135 Porto, Portugal 2Institute for Molecular Systems Biology, Swiss Federal Institute of Technology, ETH Zürich, Zürich, 8093, Switzerland 3PHRT-CPAC, ETH Zürich, Zürich, 8093, Switzerland 4SIB Swiss Institute of Bioinformatics, Lausanne, 1015, Switzerland 5Wellcome Trust-Medical Research Council, Stem Cell Institute, Cambridge CB2 2XY, UK 6Faculty of Medicine (FMUP), University of Porto, Porto, 4200319, Portugal 7Molecular Health Sciences, Department of Biology, Swiss Federal Institute of Technology, ETH Zürich, Zürich, 8093, Switzerland

* These authors contributed equally

\# Corresponding author:

Laura Montani - Switzerland; laura.montani@alumni.ethz.ch

Abbreviated Title: The remyelination proteome 


\begin{abstract}
Loss of myelin underlies the pathology of several neurological disorders of diverse etiology. CNS remyelination by adult oligodendrocyte progenitor cells (OPCs) can occur but it differs from developmental myelination carried out by neonatal OPCs. We asked whether the myelin proteome of remyelinated regions is changed. We compared the myelin proteome formed during development to the remyelination proteome attained after lysolecithin-induced demyelination in the mouse spinal cord. Mass-spectrometry analysis of iTRAQ labelled myelin protein lysates showed that the proteome of remyelination is different from that of developmental myelination, leading to profound changes in myelin protein content. Aside from known mediators of oligodendrocyte differentiation, we found proteome alterations included modulators of metabolism, cell signaling and actin cytoskeleton dynamics. Downregulating one candidate (FSCN1/Fascin1) was sufficient to partially hamper oligodendrocytes in-vitro. In summary, we identify the difference in the proteome of remyelinating oligodendrocytes as a novel potential contributor to the pathophysiology of demyelinating disorders, thus providing new potential therapeutic targets for future studies.
\end{abstract}

Keywords demyelination, remyelination, CNS, proteome, oligodendrocyte 


\section{Abbreviations}

dpi $=$ days post injury

$\mathrm{DOD}=$ days of differentiation

iTRAQ $=$ isobaric Tags for Relative and Absolute Quantitation

OPCs = Oligodendrocyte Progenitor Cells

$\mathrm{aOPCs}=$ adult OPCs

nOPCs $=$ neonatal OPCs

$\mathrm{P}(\mathrm{n})=$ Postnatal day $(\mathrm{n})$

PNS $=$ peripheral nervous system 


\section{Introduction}

In the CNS oligodendrocytes ensheath axons with myelin, a highly organized multi-membrane compacted structure crucial to ensure saltatory conduction and long term maintenance of axonal health. Thus, loss or damage of myelin is one of the major mechanisms underlying the neurodegenerative pathology observed in neurological disorders of diverse etiology, such as multiple sclerosis and leukodystrophies (Fancy et al., 2011; Franklin and Ffrench-Constant, 2017; Saab and Nave, 2017). Spontaneous remyelination carried out by adult OPCs (aOPCs) can occur in demyelinated lesions and the newly formed myelin is able to restore at least partial physiological activity (Fancy et al., 2011; Franklin and Ffrench-Constant, 2017). However, remyelination differs from the spatial and temporal organized program characteristic of developmental myelination (Fancy et al., 2011), and occurs in the presence of an active immune response (Simons et al., 2014; Miron, 2017). Although aOPCs differentiate into mature oligodendrocytes during remyelination the newly formed myelin is morphologically thinner for all but the smallest diameter myelinated axons (Fancy et al., 2011; Franklin and FfrenchConstant, 2017). Its capacity to prevent axonal degeneration in the long term has not been fully clarified (Saab and Nave, 2017). The molecular aspects associated with hypomyelination as a result of remyelination remain poorly understood, both in animal models of demyelination and in human patients (Fancy et al., 2011; Franklin and Ffrench-Constant, 2017). In particular, whether and to what extent: 1) the remyelination proteome recapitulates that of developmental myelination, and 2) altered expression patterns of proteins within the newly formed myelin contribute to ineffective remyelination, are questions which remain to be addressed. Several studies have explored changes in the proteome of demyelinated lesions in a range of models, but without discerning on the cellular origin of the observed changes (Jahn et al., 2009; Linker et al., 2009; Werner et al., 2010). Due to the complexity of the lesion environment, containing remyelinating glial cells, but strongly enriched in astrocytes and immune cells, no information on proteins which expression substantially changes in the myelin following remyelination is 
currently available. This is not trivial, as this knowledge could help identifying novel therapeutic strategies for a more efficient form of remyelination. Therefore, we addressed the above questions by specifically comparing the proteome of myelin formed after experimental demyelination (hereafter called the remyelination proteome) induced by injection of the toxin lysolecithin in the mouse spinal cord versus the proteome of myelin formed during development. Our data show that the remyelination proteome fails to recapitulate the one from developmental myelination presenting significant changes in the expression of modulators of metabolism, cell signaling and cytoskeleton remodeling proteins. We further show that downregulation of a single selected target is sufficient to partially hamper oligodendrocytes invitro.

\section{Materials and Methods}

For list of antibodies, electron microscopy, iTRAQ mass-spectrometry, qRT-PCR, immunoblotting, immunohistochemistry, and quantification methods protocols see Supplementary Materials and Methods.

\section{Animals and demyelinating lesion model}

All procedures were conducted with the approval and in accordance with the IBMC/i3S Animal Ethics Committee, the Portuguese Veterinary Office, the EU animal welfare laws, guidelines and policies, and following the ARRIVE guidelines. Wild type C57Bl/6 mice and Wistar rats of both sexes were used. Demyelination was obtained by focal injection of $1 \mu 11 \%$ lysolecithin in the ventro-lateral funiculus of the spinal cord at vertebrae level T4. See Supplementary Materials and methods.

\section{Oligodendrocyte progenitor cell isolation and oligodendrocyte cultures}

Oligodendrocyte progenitor cells were isolated from mix-glial cultures of P0-P2 Wistar Han rat brains, as described (Chen et al., 2007). See Supplementary Materials and Methods.

\section{Viral-mediated shRNA}


See Supplementary Materials and Methods.

\section{Myelinating co-cultures}

See Supplementary Materials and Methods.

\section{Experimental design}

Littermate and aged matched mice were randomly assigned to groups. No statistical method was used to predetermine sample size, but sample sizes are similar to those generally employed in the field. All quantifications were done blindly by third party concealment of treatments.

\section{Statistical analysis}

All experiments were quantified blindly to the treatment. Statistics were analyzed using GraphPad Prism vs6.01. Data were assumed to be normally distributed, but not formally tested. Variance was assumed to be equal between groups. Statistical significance was determined using an unpaired two sample Student's t-test for two group comparisons, while multiple group analysis was performed with one- or two-way analysis of variance (ANOVA) and post-hoc test as detailed in text/figures. Data show mean \pm SEM of 3 independently run experiments unless otherwise specified. Significance was set at $\mathrm{p}<0.05^{*}, \mathrm{p}<0.01 * *, \mathrm{p}<0.001 * * *$.

\section{Data availability}

The respective raw files used for iTRAQ data analysis were uploaded to a community data repository. Access credentials: ftp://MSV000085385@,massive.ucsd.edu, MSV000085385,

PWD: $\quad$ wlab@2020, $\quad$ Experiment $\quad 1$ :

[ftp://MSV000085385@massive.ucsd.edu/raw/bdamaris_M1102_002.RAW], Experiment 2:

[ftp://MSV000085385@massive.ucsd.edu/raw/bdamaris_M1102_004.RAW]

Other data are available upon request from the corresponding author. 


\section{Results}

\section{The remyelination proteome differs from the proteome of developmentally formed}

\section{myelin}

We first addressed whether remyelination carries a distinct proteome by comparing proteins expressed in remyelination- versus developmentally-formed myelin (Fig. 1A, B). We induced focal demyelination in the thoracic spinal cord of adult $\mathrm{C} 57 \mathrm{Bl} / 6$ mice by injection of lysolecithin (Fig. 1B, C and D). This model allows a temporal and spatial defined identification of a de-/re-myelinated lesion. Remyelination was largely complete at $21 \mathrm{dpi}$ (Fig. 1B, C and D). Thus, we isolated myelin by sucrose gradient centrifugation using 1-cm of spinal cord tissue dissected around the lesion site at $21 \mathrm{dpi}$ for the analysis of the remyelination proteome and from the intact myelinated spinal cord of young-adult mice for the analysis of the developmental myelin proteome (Fig. 2A). It has to be expected that a fraction of this tissue still contains normal myelin, surrounding the lesion area. Electron microscopy analysis confirmed specific enrichment in myelin membranes (Supplementary Fig. 1A). Comparison by SDS-PAGE and silver staining of total protein lysates obtained from myelin membranes isolated from remyelinated versus developmentally myelinated spinal cord tissue revealed the presence of numerous different bands (Supplementary Fig. 2A, arrows). To map the differentially regulated proteins, 2 independent biological samples per condition (consisting of spinal cord tissue pooled from 6-8 mice) were obtained and protein lysates labelled with iTRAQ reagents, followed by mass spectrometry and bioinformatic analysis (Fig. 2A, Supplementary Fig. 2B, C, and D). Applying an arbitrary 1.2-fold change threshold, we identified 103 proteins differentially expressed in myelin formed during remyelination when compared to a same total amount of myelin formed during development (Table 1, Supplementary Table 1). In setting this fold threshold, we took into consideration that differences in protein expression could be underestimated, due to remaining normal myelin in the dissected lesion-containing tissue. The great majority $(97.1 \%)$ of regulated proteins showed decreased expression in the remyelination 
proteome compared to the naïve myelin proteome (Table 1, Supplementary Table 1). Only exceptions seem to be non-myelin proteins, probably derived from traces of contaminating blood (Hemoglobin-a and -b, Apolipoprotein A-II). Our results present relative changes, thus a strong fold change increase in a protein which is present in traces may have a far less biological significance than a small fold change decrease in a protein which is present at a much higher absolute quantity in the purified myelin samples (e.g. myelin specific proteins). We clustered the regulated proteins into functional categories by automated Panther analysis (www.pantherdb.org) (Fig. 2B, Table 1). Metabolism-related, cytoskeletal and signaling proteins were the main affected functional categories (Fig. 2B, Table 1). The remyelination proteome further revealed a myelin response (Fig. 2B, Table 1), characterized by lower expression of a subset of myelin specific proteins: MOG, MOBP, CNPase, and MAG. On the contrary, MBP showed comparable abundance in the remyelination versus the naïve myelin proteome, as also confirmed by immunoblotting (Supplementary Fig. 3 A and 4A). We also found dysregulation of proteins involved in fatty acid and cholesterol metabolism (Fig. 2B, Table 1). Literature mining revealed that some of these dysregulated targets were previously identified in the EAE mouse model and/or in plaques of multiple sclerosis patients. These included: Stathmin, Creatine-kinaseB, Sirtuin2, CRMP2 and 3 (Table 1, Supplementary Table 1) (Liu et al., 2005; Jastorff et al., 2009).

In summary our proteomic analysis revealed that CNS remyelination leads to a distinct myelin protein composition, which does not fully recapitulate the one present in naïve developmentally-formed myelin.

\section{Altered expression of cytoskeleton remodeling proteins in the remyelination}

\section{proteome}

We next assessed whether observed differences in expression of proteins in the remyelination compared to the developmental myelin proteome could reflect a failure in molecular hubs critical for efficient myelination. We ran an unbiased Metacore (https://portal.genego.com/) 
enrichment analysis, which revealed inhibition of pathways and processes involved in the development of oligodendrocytes and in cytoskeleton remodeling (Fig. 2C, D). Modulation of actin- and tubulin-binding proteins downstream of Rho-GTPase signaling is critical towards efficient developmental myelination (Feltri et al., 2008). In particular, tight regulation of actin dynamics is crucial in driving myelin growth (Nawaz et al., 2015; Zuchero et al., 2015). This led us to focus onto this proteins cluster.

Our analysis found the expression of RhoGDI, a key regulator of Rho-GTPase signaling, and of two of the main modulators of actin dynamics, namely CFL1/cofilin1 and FSCN1/Fascin1 to be downregulated in the remyelination proteome (Table 1 and Supplementary Table 1). Downregulation of FSCN1 (Fig. 3A and Supplementary 4C) and RhoGDI (Supplementary Fig. 3B and 4B) was further confirmed by immunoblotting. The phosphorylation levels of Ser-3, which regulate the function of CFL1 on actin were also diminished (Supplementary Fig. 3B and 4B). Our data show that these modulators of actin cytoskeletal dynamics in the remyelination proteome were significantly downregulated compared to the naïve myelin proteome.

\section{Downregulating a single identified target is sufficient to partially impair}

\section{oligodendrocytes in-vitro}

Our screening identified changed expression of a plethora of proteins in the remyelination proteome. It is possible that the incomplete myelin formation of remyelination (thinner sheaths) in the lesioned CNS is the result of the synergistic effects of these concomitant changes. As an example, we reasoned that the downregulation in actin cytoskeleton-remodeling proteins might alter the dynamic of actin filament assembly/disassembly necessary for correct oligodendrocyte differentiation and/or myelin formation. To test if and to which degree dysregulation of a single identified target may impact on oligodendrocytes, we selected to address in-vitro the effect of downregulating the actin modulator FSCN1, which is transcribed at high levels in oligodendrocytes (http://www.brainrnaseq.org/). 
We cultured neonatal rat-derived OPCs and induced them to differentiate in-vitro into mature oligodendrocytes. Quantitative real-time PCR confirmed that Fscn1 transcription increased during in-vitro differentiation (Fig. 3B), and immunocytochemistry analysis showed FSCN1 protein to be expressed in oligodendrocytes (Olig2+), also at their mature MBP+ stage (Fig. 3C). We next studied the consequences of knocking down FSCN1 expression in-vitro in differentiating oligodendrocytes. We selected a validated shRNA sequence targeting Fscn1, based on our confirmation of its ability to decrease its expression (Fig. 3D). To test for a potential function of FSCN1 in regulating the morphological differentiation of oligodendrocytes, transduced cultured oligodendrocytes were categorized in 4 different classes based on the complexity of the branching of their processes, as previously described (Baer et al., 2009) (Fig. 3E). At 0 DOD Fscn1 shRNA-transfected OPCs showed longer processes (Fig. $3 \mathrm{~F})$. This observation was confirmed by Sholl analysis, with process length up to $>150 \mu \mathrm{m}$ in Fscn1 shRNA-transduced cells (Fig. 3G) compared to $\sim 100 \mu \mathrm{m}$ in control DsRed2 shRNAtransduced oligodendrocytes (Fig. 3G). Despite this early effect, FSCN1 knock down did not significantly affect oligodendrocyte differentiation (Fig. 3H). In fact, the percentage of cells assigned to four categories of morphological differentiation at 0,3 and 6 DOD in-vitro in both control and Fscn1 shRNA-transduced cells were not significantly different (Fig. 3H). Furthermore, at 6 DOD no significant differences were found between controls and FSCN1 knockdown OL concerning the percentage of mature (MBP+ lamellae-forming) oligodendrocytes (Supplementary Fig. 5A), or in their capacity of forming myelin-like sheaths (Supplementary Fig. 5B).

Next, to investigate whether reducing FSCN expression impacted on the capacity of oligodendrocytes to myelinate axons, we plated FACS-sorted GFP-expressing Fscn1 shRNAtransduced oligodendrocytes (48 hours post infection) onto purified naïve dorsal root ganglia neurons. After 18 days in culture, oligodendrocytes transduced with control shRNA had myelinated axons forming several myelin internodes (Fig. 3I). In contrast, oligodendrocytes in 
which FSCN1 had been knocked down displayed a lower number of internodes per cell (Fig. 3I and 3J), with a slightly increased length (Supplementary Fig. 5C).

In summary, our data show that knocking down a single target - altered in the remyelination proteome compared to the myelination proteome - may be sufficient to impact upon oligodendrocyte normal characteristics.

\section{Discussion}

In this study, we assessed whether the remyelination proteome differs from the developmental myelin proteome. Remyelinating tissue contains high density of astrocytes, microglia and lymphocytes that proliferate and get activated at the lesion site. Thus, using as input myelin contaminated with membranes from these cells is likely to mostly identify the upregulation of markers for gliosis in a proteomics comparison of lesioned versus intact tissue. A contamination with astrocytes, microglia and lymphocytes and their respective proteomes was prevented by applying a myelin-isolation protocol. No marker for gliosis was found to be upregulated. Our proteomic analysis of myelin isolated from lesioned versus intact tissue showed that the proteome of myelin formed following remyelination is distinct from that of the myelin formed during development. Based on these results and functional in-vitro assays, we propose that changes in the expression of key modulators of actin cytoskeleton dynamics might contribute to restrict the extent of remyelination.

Several lines of evidence established that regulation of actin dynamics is key in driving oligodendrocyte differentiation and myelination during development (Nawaz et al., 2015; Samanta and Salzer, 2015; Zuchero et al., 2015; Shao et al., 2017; Azevedo et al., 2018). Our data provide evidence that actin binding proteins are dysregulated in myelin formed following de-/re-myelination and that inefficient expression of a single actin bundling protein, FSCN1, is sufficient to at least partially impact upon oligodendrocytes normal morphology in-vitro. This 
shows that our approach is suitable for a first identification of novel potential therapeutic targets, which will require further validation in-vivo in future studies. Nonetheless, the changes observed upon in-vitro knock down of a single target, i.e. FSCN1, were not dramatic. This lead us to conceive that inefficient remyelination is likely to be driven by the concomitant dysregulation of several proteins. Identifying the molecular mechanisms leading to their lower expression in remyelinating oligodendrocytes remains a goal for future studies.

We were surprised to find downregulation of major myelin proteins in the remyelination proteome compared to the naïve myelin proteome. We cannot rule out that the presence of aOPC-derived Schwann cell remyelination (Zawadzka et al., 2010) may partially account for the observed lower expression of CNS myelin-specific proteins, MOG and MOBP. However, this could not explain the decrease in MAG and CNPase, also expressed by Schwann cells. MBP, another major myelin protein expressed by both oligodendrocytes and Schwann cells, showed no significant difference in its total expression in the remyelination versus the myelin naïve proteome. Nonetheless immunoblotting revealed diverse level of expression of its different isoforms. Our data may indicate dysregulation of specific myelin proteins following remyelination in the applied lesion model. This finding was unexpected as these proteins are critical to maintain the highly compacted organization of myelin membranes (Bakhti et al., 2014), thus highlighting the importance of future studies addressing the long term stability of remyelinated profiles. It was surprising that $97 \%$ of the differentially expressed proteins identified displayed lower expression (by more than 1.2 fold) in remyelinated membranes compared to myelinated membranes. It must be considered that the samples may be in different "developmental" stages, i.e. P21 myelin is fully mature/homeostatic whereas 21 dpi myelin is still undergoing maturation. This could account for the observed diverse expression of myelin specific proteins. Applying a similar experimental approach to later time points, in future studies, will allow to address if and when myelin formed in the remyelination process is capable of reaching maturity. 
In the course of our study we found that proteins involved in the metabolism of cholesterol and fatty acids showed a diverse expression in the remyelination versus the naïve myelin proteome. Myelination relies on the production of a massive amount of cell membranes which contain a high percentage of lipids. Accordingly, both fatty acid synthesis and cholesterol are critical for myelination during development (Saher et al., 2005; Schmitt et al., 2015; Camargo et al., 2017; Montani et al., 2018; Dimas et al., 2019). It is therefore conceivable that an impairment in lipid synthesis and/or transport could contribute in restricting myelin growth during remyelination, an aspect that warrants further attention. We cannot currently exclude that control versus remyelinated samples may present a diverse quality of myelin enrichment in the myelin isolated fraction, e.g. due to a diverse solubility of myelin membranes from remyelinated tissue due to a different lipid composition. This is an interesting and complex aspect which remains to be further addressed.

\section{Acknowledgments}

We thank members of the Relvas lab for discussion and the Scientific Platforms of IBMC/i3S for excellent technical support.

\section{Funding}

This project was funded by the FP7-People-Program (AXOGLIA-633792) and the Portuguese Science\&Technology Foundation (FCT/PTDC-SAU-NEU/2008/090077), LM by a Marie-

Curie fellowship (AXOGLIA-633792), JPF/MMA/AIS/HSD by an FCT fellowship (BPD/SFHR/34834/2007;SFRH/BD/90301/2012;SFRH/BPD/79417/2011;SFRH/BPD/90268/ 2012), RJMF and CZ by a grant from the UK Multiple Sclerosis Society and a core support grant from the Wellcome-Trust and MRC to the Wellcome-Trust - Medical Research Council Cambridge Stem Cell Institute.

\section{Competing Interests}

The authors report no competing interests. 


\section{Supplementary Material}

Supplementary material is available as separate .pdf file.

\section{References}

Azevedo MM, Domingues HS, Cordelieres FP, Sampaio P, Seixas AI, Relvas JB. Jmy regulates oligodendrocyte differentiation via modulation of actin cytoskeleton dynamics. Glia 2018.

Baer AS, Syed YA, Kang SU, Mitteregger D, Vig R, Ffrench-Constant C, et al. Myelinmediated inhibition of oligodendrocyte precursor differentiation can be overcome by pharmacological modulation of Fyn-RhoA and protein kinase C signalling. Brain 2009; 132(Pt 2): $465-81$.

Bakhti M, Aggarwal S, Simons M. Myelin architecture: zippering membranes tightly together. Cell Mol Life Sci 2014; 71(7): 1265-77.

Camargo N, Goudriaan A, van Deijk AF, Otte WM, Brouwers JF, Lodder H, et al. Oligodendroglial myelination requires astrocyte-derived lipids. PLoS Biol 2017; 15(5): e1002605.

Chen Y, Balasubramaniyan V, Peng J, Hurlock EC, Tallquist M, Li J, et al. Isolation and culture of rat and mouse oligodendrocyte precursor cells. Nat Protoc 2007; 2(5): 1044-51.

Dimas P, Montani L, Pereira JA, Moreno D, Trotzmuller M, Gerber J, et al. CNS myelination and remyelination depend on fatty acid synthesis by oligodendrocytes. Elife 2019; 8 .

Fancy SP, Chan JR, Baranzini SE, Franklin RJ, Rowitch DH. Myelin regeneration: a recapitulation of development? Annu Rev Neurosci 2011; 34: 21-43.

Feltri ML, Suter U, Relvas JB. The function of RhoGTPases in axon ensheathment and myelination. Glia 2008; 56(14): 1508-17.

Franklin RJM, Ffrench-Constant C. Regenerating CNS myelin - from mechanisms to experimental medicines. Nat Rev Neurosci 2017; 18(12): 753-69.

Jahn O, Tenzer S, Werner HB. Myelin proteomics: molecular anatomy of an insulating sheath. Mol Neurobiol 2009; 40(1): 55-72. 
Jastorff AM, Haegler K, Maccarrone G, Holsboer F, Weber F, Ziemssen T, et al. Regulation of proteins mediating neurodegeneration in experimental autoimmune encephalomyelitis and multiple sclerosis. Proteomics Clin Appl 2009; 3(11): 1273-87.

Linker RA, Brechlin P, Jesse S, Steinacker P, Lee DH, Asif AR, et al. Proteome profiling in murine models of multiple sclerosis: identification of stage specific markers and culprits for tissue damage. PLoS One 2009; 4(10): e7624.

Liu A, Stadelmann C, Moscarello M, Bruck W, Sobel A, Mastronardi FG, et al. Expression of stathmin, a developmentally controlled cytoskeleton-regulating molecule, in demyelinating disorders. J Neurosci 2005; 25(3): 737-47.

Miron VE. Microglia-driven regulation of oligodendrocyte lineage cells, myelination, and remyelination. J Leukoc Biol 2017; 101(5): 1103-8.

Montani L, Pereira JA, Norrmen C, Pohl HBF, Tinelli E, Trotzmuller M, et al. De novo fatty acid synthesis by Schwann cells is essential for peripheral nervous system myelination. J Cell Biol 2018.

Nawaz S, Sanchez P, Schmitt S, Snaidero N, Mitkovski M, Velte C, et al. Actin filament turnover drives leading edge growth during myelin sheath formation in the central nervous system. Dev Cell 2015; 34(2): 139-51.

Saab AS, Nave KA. Myelin dynamics: protecting and shaping neuronal functions. Curr Opin Neurobiol 2017; 47: 104-12.

Saher G, Brugger B, Lappe-Siefke C, Mobius W, Tozawa R, Wehr MC, et al. High cholesterol level is essential for myelin membrane growth. Nat Neurosci 2005; 8(4): 468-75.

Samanta J, Salzer JL. Myelination: actin disassembly leads the way. Dev Cell 2015; 34(2): 12930.

Schmitt S, Castelvetri LC, Simons M. Metabolism and functions of lipids in myelin. Biochimica et biophysica acta 2015; 1851(8): 999-1005. 
Shao Z, Lee X, Huang G, Sheng G, Henderson CE, Louvard D, et al. LINGO-1 Regulates Oligodendrocyte Differentiation through the Cytoplasmic Gelsolin Signaling Pathway. J Neurosci 2017; 37(12): 3127-37.

Simons M, Misgeld T, Kerschensteiner M. A unified cell biological perspective on axon-myelin injury. J Cell Biol 2014; 206(3): 335-45.

Werner SR, Saha JK, Broderick CL, Zhen EY, Higgs RE, Duffin KL, et al. Proteomic analysis of demyelinated and remyelinating brain tissue following dietary cuprizone administration. $\mathrm{J}$ Mol Neurosci 2010; 42(2): 210-25.

Zawadzka M, Rivers LE, Fancy SP, Zhao C, Tripathi R, Jamen F, et al. CNS-resident glial progenitor/stem cells produce Schwann cells as well as oligodendrocytes during repair of CNS demyelination. Cell Stem Cell 2010; 6(6): 578-90.

Zuchero JB, Fu MM, Sloan SA, Ibrahim A, Olson A, Zaremba A, et al. CNS myelin wrapping is driven by actin disassembly. Dev Cell 2015; 34(2): 152-67.

\section{Figure legends}

Figure 1. Experimental design. (A) Diagram illustrating the experimental design for the mapping of the changes in the proteome of myelin from the spinal cord following experimental de- and re-myelination. The proteome of myelin formed by aOPCs-derived oligodendrocytes, encasing axons following lysolecithin-induced focal demyelination, is compared to that of myelin formed by nOPCs-derived oligodendrocytes during development. (B) Timeline of lysolecithin-induced focal demyelination in the thoracic spinal cord (vertebrae level T4) and subsequent remyelination. Analyzed time-points are shown. (C) Representative toluidine blue staining of the ventral thoracic spinal cord at vertebrae level T4: postnatal day (P) 21 with axons invested by myelin, 5 days post-lysolecithin-injection (dpi) with a ventrolateral focal demyelinated lesion, and 21 dpi with axons reinvested by thinner myelin following remyelination. $n=3$ mice observed per each time point. Scale bar: $250 \mu \mathrm{m}$ (D) Representative 
micrographs depicting the morphology of the ventrolateral white matter by electron transmission microscopy: P21 with axons invested by myelin, 5 dpi with demyelinated axons in the lesion area (examples false colored in orange, examples of axons spared from demyelination indicated by asterisks), and 21 dpi with axons encased by thinner myelin following remyelination (examples indicated by arrow heads). $n=3$ mice observed per each time point. Scale bar: $2.5 \mu \mathrm{m}$. OPCs $=$ oligodendrocyte progenitor cells, dpi $=$ days post injection, $\mathrm{P}=$ postnatal day, $\mathrm{w}=$ weeks, $\mathrm{GM}=$ grey matter, $\mathrm{WM}=$ white matter.

\section{Figure 2. Analysis of the remyelination proteome reveals dysregulation of the cytoskeletal}

machinery. (A) iTRAQ experimental pipeline. $1 \mathrm{~cm}$ of spinal cord tissue around the lesion site (vertebrae level T4) was dissected from P21 naïve and 21 dpi C57Bl/6 mice for subsequent isolation of myelin on a sucrose gradient. Protein extracts were further processed for iTRAQ labelling and subsequent mass-spectrometry identification and relative quantification. (B) Diagram depicting percentage of dysregulated proteins based on assigned functional categories. $n=2$ independent experiments, each with an independent set of pooled spinal cords for both 21 dpi and P21, each set from 6-8 mice (1 $\mathrm{cm}$ of tissue around the lesion site or $1 \mathrm{~cm}$ from intact spinal cord tissue at the same thoracic spinal cord level). (C, D) Cytoskeletal-related Process Networks (C) and Pathway Maps (D) dysregulated in the remyelination proteome compared to the naïve myelin proteome, as identified by Metacore (version 6.29) enrichment analysis based on those proteins significantly downregulated in both independent experiments. Data points represent the total count of regulated proteins per each category (one value per category as output). Bar graphs represents the total - $\log \mathrm{P}$ value per each category (one value per category as output).

Figure 3. Consequences of decreasing FSCN1 expression in oligodendrocytes in-vitro. (A) Lower FSCN1 expression in newly adult-formed myelin following demyelination (21 dpi) compared to developmentally formed myelin (P21), shown by immunoblotting. Same amount of total proteins loaded (10 $\mu \mathrm{g}$ per lane). Due to their dysregulation at 21 dpi compared to P21, 
commonly used housekeeper proteins (GAPDH, Actin and Tubulin, see Table 1 and Supplementary Table 1) could not be used as loading control. CRMP-5 was not identified as regulated in the proteomic screening, and it is shown as reference protein for loading control as not being detected as regulated by immunoblotting. $n=2$ independent sets of pooled spinal cords for both $21 \mathrm{dpi}$ and $\mathrm{P} 21$, each from 8 mice $(1 \mathrm{~cm}$ of tissue around the lesion site or $1 \mathrm{~cm}$ from intact spinal cord tissue at the same thoracic spinal cord level). (B) Graph of qRT-PCR analysis for Fscn1 at day of differentiation (DOD) 0, 1 and 3 in OPC-differentiating cultures. Data normalized to Ywhaz and to the mean of DOD 0. Data points represent $n=3$ experiments (1-way Anova with Tukey's multiple comparisons test DOD 0 vs. DOD $1 d f=2, q=9.489$, DOD 0 vs DOD $3 d f=2, q=7.586), * p<0.05, \# p=0.0597$. (C) Representative immunostaining of differentiating-OPC cultures at 0 and 6 DOD. FSCN1 expression was detected in all Olig2+ oligodendrocytes already at 0 DOD. Note the prominent FSCN1 expression in fully mature promyelinating $(\mathrm{MBP}+)$ oligodendrocytes at 6 DOD. $n=3$ observed experiments. Scale bar: $20 \mu \mathrm{m}$. (D) Cropped FSCN1 immunoblot with $\alpha$-tubulin and GAPDH detected in the same membrane as loading controls. Quantification is shown in Supplementary Fig. 3C. From protein lysates obtained from OPCs infected with Fscn1 shRNA or DsRed2 shRNA, GFP+ sorted 48 hours following infection. Representative of $n=3$ independent experiments. Full-length immunoblot in Supplementary Fig. 4. (E, F) Scheme of stage of differentiation along the oligodendrocyte-lineage (E) and representative immunostaining images (F) of cultures of GFP+ sorted Olig2+ OPCs treated with Fscn1 shRNA or control DsRed2 shRNA at 0, 3 and 6 DOD. Scale bar: $50 \mu \mathrm{m}$. (G) Graphic representation of Sholl Analysis' results depicting the number of processes intersecting a radius at increasing distances from the cell body of oligodendrocytes. Data points represent $n=3$ independent experiments (2-way Anova with Sidak's multiple comparisons test Fscn1 shRNA vs. DsRed2 shRNA, $p<$ $\left.0.0001, F_{10,40}=31.82\right)$. (H) Corresponding bar graphs with quantification of percentage of cells assigned to the four different stages of oligodendrocyte differentiation, from OPC infected with 
Fscn1 shRNA or control DsRed2 shRNA, GFP+ sorted and differentiated in culture for 0,3 and 6 DOD. Data points represent $n=3$ independent experiments (two-way Anova with Sidak's multiple comparisons test, Fscn1 shRNA vs. DsRed 2 shRNA, DOD 0 stage I $p=0.1398$, stage II $p=0.3005$, stage III $p=0.9872$, stage IV $p>0.9999$, DOD 3 stage I $p=0.9998$, stage II $p=$ 0.9856, stage III $p=0.9785$. stage IV $p>0.9999$, DOD 6 stage I $p<0.9999$, stage II $p=0.3060$, stage III $p=0.9282$, stage IV $p=0.7410$ ). (I) Representative immunostaining of myelinating oligodendrocytes (MBP+) transduced with Fscn1 shRNA or DsRed2 shRNA as control, grown upon DRG neurites $(\mathrm{NF}+)$. Note the higher number of internodes formed by control oligodendrocytes (internodes traced in yellow). $n=2$ independent experiments. Scale bar: 50 $\mu \mathrm{m}$. (J) Corresponding bar graph showing the Internode formation index (= number of internodes per oligodendrocyte, normalized to the mean of the control set to 100) (unpaired two-tailed two sample Student's t-test, Fscn1 shRNA vs. DsRed2 shRNA $p<0.0001, t=7.282$ ), *** $\mathrm{p}<0.001 .9$ to 16 cells analyzed per condition and experiment, in 2 independent experiments. Bars represent mean \pm SEM. DOD $=$ days of differentiation, OPCs $=$ oligodendrocyte progenitor cells. 
A

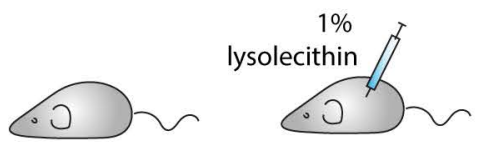

\section{C57BI/6}

P21 näive

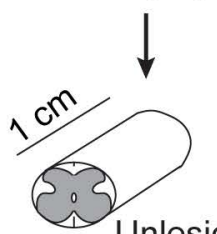

Unlesioned Spinal cord

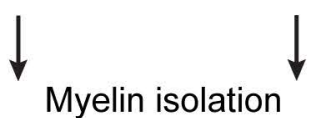
on sucrose gradient

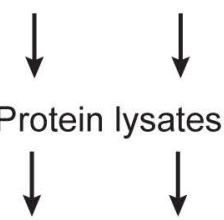

Reduced, alkylated, digested Labelled with ITRAQ reagents
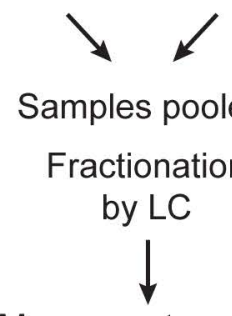

Mass spectrometry analysis
Proteins assigned to

Functional Categories

(\%)

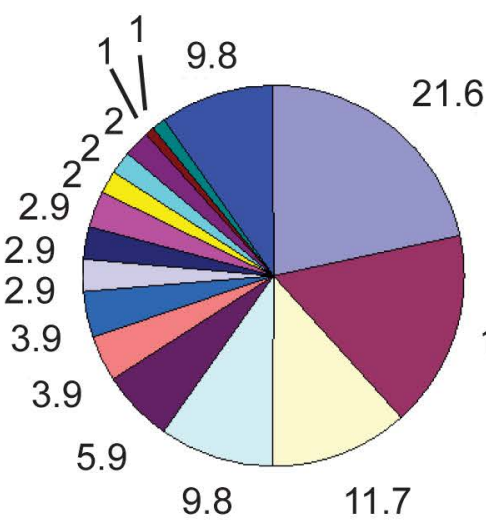

Myelin 21dpi vs. P21

(103 regulated proteins) $\square$ 1. Metabolism

2. Cytoskeleton

$\square$ 3. Signalling

$\square$ 4. Mitochondrial

5. Stress response

$\square$ 6. Myelin response

$\square$ 7. Neuronal

$\square$ 8. Endoplasmic reticulum

9. FAs/Chol metabolism

$\square$ 10. Ubiquitination

11. Channels

12. Ribosomal

13. Transport/vesicles

14. Cell adhesion

15. Immune response

16. Others
C

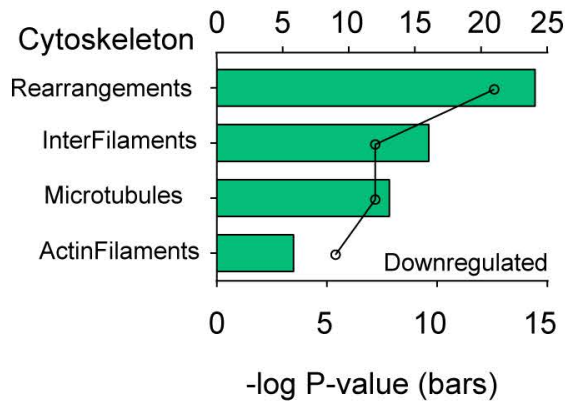

D

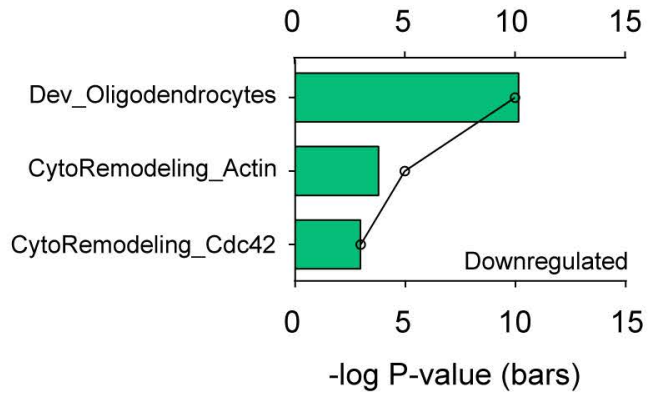


Table I. Proteins deregulated following remyelination, as identified by iTRAQ labelling followed by LCMS/MS analysis.

\section{MYELIN (21dpi versus P21)}

\begin{tabular}{|c|c|c|c|}
\hline Protein & Mean Ratio & Standard Error & $\begin{array}{l}\text { Fold } \\
\text { Change }\end{array}$ \\
\hline \multicolumn{4}{|l|}{ CELL ADHESION } \\
\hline Neural cell adhesion molecule 1 & 0.54 & 0.12 & -1.85 \\
\hline \multicolumn{4}{|l|}{ CHANNELS } \\
\hline $\begin{array}{l}\text { Sodium/potassium-transporting ATPase subunit } \\
\text { alpha-3 }\end{array}$ & 0.41 & 0.10 & -2.44 \\
\hline $\begin{array}{l}\text { Sodium/potassium-transporting ATPase subunit } \\
\text { beta-1 }\end{array}$ & 0.42 & 0.11 & -2.38 \\
\hline \multicolumn{4}{|l|}{ CYTOSKELETON } \\
\hline Actin, cytoplasmic 2 & 0.50 & 0.04 & -2.00 \\
\hline Tubulin alpha-1A chain & 0.36 & 0.01 & -2.78 \\
\hline Fascin & 0.40 & 0.17 & -2.50 \\
\hline Cofilin-1 & 0.43 & 0.04 & -2.33 \\
\hline Spectrin alpha chain, brain & 0.45 & 0.08 & -2.22 \\
\hline Stathmin-2 & 0.51 & 0.03 & -1.96 \\
\hline Tubulin polymerization-promoting protein & 0.51 & 0.10 & -1.96 \\
\hline Tubulin beta- 4 chain & 0.52 & 0.08 & -1.92 \\
\hline Tubulin beta- 5 chain & 0.59 & 0.08 & -1.69 \\
\hline Actin, alpha skeletal muscle & 0.60 & 0.01 & -1.67 \\
\hline Tubulin beta-2A chain & 0.66 & 0.04 & -1.52 \\
\hline Alpha-internexin & 0.71 & 0.05 & -1.41 \\
\hline \multicolumn{4}{|l|}{ ENDOPLASMIC RETICULUM } \\
\hline Calreticulin & 0.35 & 0.08 & -2.86 \\
\hline Transitional endoplasmic reticulum ATPase & 0.49 & 0.05 & -2.04 \\
\hline \multicolumn{4}{|l|}{ FATTY ACID AND CHOLESTEROL METABOLISM } \\
\hline Fatty acid-binding protein, brain & 0.49 & 0.11 & -2.04 \\
\hline Apolipoprotein A-II & 1.68 & 0.34 & 1.68 \\
\hline \multicolumn{4}{|l|}{ METABOLISM } \\
\hline Isocitrate dehydrogenase [NADP] cytoplasmic & 0.32 & 0.09 & -3.13 \\
\hline $\begin{array}{l}\text { Ectonucleotide } \\
\text { pyrophosphatase/phosphodiesterase family } \\
\text { member } 6\end{array}$ & 0.52 & 0.08 & -1.92 \\
\hline L-lactate dehydrogenase B chain & 0.52 & 0.05 & -1.92 \\
\hline Fructose-bisphosphate aldolase C & 0.58 & 0.04 & -1.72 \\
\hline Phosphoglycerate mutase 1 & 0.58 & 0.04 & -1.72 \\
\hline Phosphoglycerate kinase 1 & 0.58 & 0.04 & -1.72 \\
\hline $\begin{array}{l}\text { Platelet-activating factor acetylhydrolase IB subunit } \\
\text { beta }\end{array}$ & 0.60 & 0.11 & -1.67 \\
\hline Alpha-enolase & 0.61 & 0.03 & -1.64 \\
\hline Glucose-6-phosphate isomerase & 0.61 & 0.05 & -1.64 \\
\hline Lactoylglutathione lyase & 0.62 & 0.03 & -1.61 \\
\hline Glyceraldehyde-3-phosphate dehydrogenase & 0.63 & 0.03 & -1.59 \\
\hline Pyruvate kinase isozymes M1/M2 & 0.64 & 0.04 & -1.56 \\
\hline Adenylate kinase isoenzyme 1 & 0.64 & 0.06 & -1.56 \\
\hline
\end{tabular}




\begin{tabular}{|c|c|c|c|}
\hline Protein & Mean Ratio & Standard Error & $\begin{array}{c}\text { Fold } \\
\text { Change }\end{array}$ \\
\hline Aspartate aminotransferase, cytoplasmic & 0.64 & 0.11 & -1.56 \\
\hline Gamma-enolase & 0.66 & 0.05 & -1.52 \\
\hline Glutathione S-transferase Mu 1 & 0.66 & 0.07 & -1.52 \\
\hline Glutamine synthetase & 0.68 & 0.09 & -1.47 \\
\hline Glutamate dehydrogenase 1 , mitochondrial & 0.69 & 0.14 & -1.45 \\
\hline Triosephosphate isomerase & 0.71 & 0.02 & -1.41 \\
\hline Malate dehydrogenase, cytoplasmic & 0.71 & 0.04 & -1.41 \\
\hline Protein disulfide-isomerase $\mathrm{A} 3$ & 0.40 & 0.09 & -2.50 \\
\hline Peptidyl-prolyl cis-trans isomerase A & 0.45 & 0.05 & -2.22 \\
\hline Transketolase & 0.46 & 0.06 & -2.17 \\
\hline Fructose-bisphosphate aldolase $\mathrm{A}$ & 0.48 & 0.04 & -2.08 \\
\hline \multicolumn{4}{|l|}{ MITOCHONDRIAL } \\
\hline Creatine kinase U-type, mitochondrial & 0.44 & 0.17 & -2.27 \\
\hline Aspartate aminotransferase, mitochondrial & 0.51 & 0.11 & -1.96 \\
\hline Malate dehydrogenase, mitochondrial & 0.55 & 0.04 & -1.82 \\
\hline Aconitate hydratase, mitochondrial & 0.57 & 0.05 & -1.75 \\
\hline Peroxiredoxin-5, mitochondrial & 0.59 & 0.21 & -1.69 \\
\hline 4-aminobutyrate aminotransferase, mitochondrial & 0.63 & 0.15 & -1.59 \\
\hline $60 \mathrm{kDa}$ heat shock protein, mitochondrial & 0.49 & 0.05 & -2.04 \\
\hline ATP synthase subunit beta, mitochondrial & 0.39 & 0.08 & -2.56 \\
\hline \multicolumn{4}{|l|}{ MYELIN } \\
\hline NAD-dependent deacetylase sirtuin-2 & 0.34 & 0.15 & -2.94 \\
\hline Myelin-associated glycoprotein & 0.35 & 0.19 & -2.86 \\
\hline 2',3'-cyclic-nucleotide 3'-phosphodiesterase & 0.40 & 0.04 & -2.50 \\
\hline Myelin-associated oligodendrocyte basic protein & 0.52 & 0.11 & -1.92 \\
\hline Myelin-oligodendrocyte glycoprotein & 0.53 & 0.12 & -1.89 \\
\hline \multicolumn{4}{|l|}{ NEURON ENRICHED } \\
\hline Tubulin beta- 3 chain & 0.71 & 0.04 & -1.41 \\
\hline Microtubule-associated protein 2 & 0.53 & 0.08 & -1.89 \\
\hline Neurofilament light polypeptide & 0.62 & 0.04 & -1.61 \\
\hline Neurofilament heavy polypeptide & 0.64 & 0.04 & -1.56 \\
\hline Brain acid soluble protein 1 & 0.35 & 0.09 & -2.86 \\
\hline Syntaxin-binding protein 1 & 0.55 & 0.15 & -1.82 \\
\hline Complexin-1 & 0.69 & 0.03 & -1.45 \\
\hline Transgelin-3 & 0.57 & 0.06 & -1.75 \\
\hline \multicolumn{4}{|l|}{ PROTEIN SYNTHESIS } \\
\hline Elongation factor 1-alpha 1 & 0.30 & 0.08 & -3.33 \\
\hline Elongation factor 1-alpha 2 & 0.52 & 0.05 & -1.92 \\
\hline Eukaryotic translation initiation factor $5 \mathrm{~A}-1$ & 0.45 & 0.04 & -2.22 \\
\hline \multicolumn{4}{|l|}{ SIGNALLING } \\
\hline 14-3-3 protein zeta/delta & 0.55 & 0.04 & -1.82 \\
\hline Calretinin & 0.74 & 0.09 & -1.35 \\
\hline Creatine kinase B-type & 0.80 & 0.03 & -1.25 \\
\hline 14-3-3 protein epsilon & 0.38 & 0.04 & -2.63 \\
\hline Ras-related protein Rap-1b & 0.40 & 0.08 & -2.50 \\
\hline
\end{tabular}




\begin{tabular}{|c|c|c|c|}
\hline Protein & Mean Ratio & Standard Error & $\begin{array}{l}\text { Fold } \\
\text { Change }\end{array}$ \\
\hline Dihydropyrimidinase-related protein 3 & 0.43 & 0.04 & -2.33 \\
\hline Rho GDP-dissociation inhibitor 1 & 0.47 & 0.1 & -2.13 \\
\hline 14-3-3 protein gamma & 0.50 & 0.03 & -2.00 \\
\hline Dihydropyrimidinase-related protein 2 & 0.50 & 0.05 & -2.00 \\
\hline $\begin{array}{l}\text { Serine/threonine-protein phosphatase } 2 \mathrm{~A} 65 \mathrm{kDa} \\
\text { regulatory subunit A alpha isoform }\end{array}$ & 0.52 & 0.11 & -1.92 \\
\hline Rab GDP dissociation inhibitor alpha & 0.55 & 0.11 & -1.82 \\
\hline Nucleoside diphosphate kinase A & 0.62 & 0.08 & -1.61 \\
\hline Phosphatidylethanolamine-binding protein 1 & 0.64 & 0.11 & -1.56 \\
\hline Nucleoside diphosphate kinase B & 0.70 & 0.06 & -1.43 \\
\hline \multicolumn{4}{|l|}{ STRESS RESPONSE } \\
\hline Peroxiredoxin-1 & 0.45 & 0.05 & -2.22 \\
\hline Peroxiredoxin-2 & 0.49 & 0.06 & -2.04 \\
\hline Heat shock cognate $71 \mathrm{kDa}$ protein & 0.51 & 0.03 & -1.96 \\
\hline Heat shock protein HSP 90-alpha & 0.53 & 0.06 & -1.89 \\
\hline $10 \mathrm{kDa}$ heat shock protein, mitochondrial & 0.59 & 0.06 & -1.69 \\
\hline Superoxide dismutase [Cu-Zn] & 0.79 & 0.03 & -1.27 \\
\hline Heat shock protein HSP 90-beta & 0.46 & 0.05 & -2.17 \\
\hline \multicolumn{4}{|l|}{ UBIQUITINATION } \\
\hline Ubiquitin & 0.40 & 0.05 & -2.50 \\
\hline Ubiquitin-conjugating enzyme E2 L3 & 0.44 & 0.16 & -2.27 \\
\hline Ubiquitin-like modifier-activating enzyme 1 & 0.52 & 0.07 & -1.92 \\
\hline \multicolumn{4}{|l|}{ OTHERS } \\
\hline Macrophage migration inhibitory factor & 0.47 & 0.09 & -2.13 \\
\hline Cytoplasmic dynein 1 heavy chain 1 & 0.53 & 0.11 & -1.89 \\
\hline Clathrin heavy chain 1 & 0.42 & 0.04 & -2.38 \\
\hline Serotransferrin & 0.40 & 0.06 & -2.50 \\
\hline Visinin-like protein 1 & 0.41 & 0.03 & -2.44 \\
\hline $\begin{array}{l}\text { Guanine nucleotide-binding protein } \mathrm{G}(\mathrm{o}) \text { subunit } \\
\text { alpha }\end{array}$ & 0.42 & 0.08 & -2.38 \\
\hline Annexin A6 & 0.51 & 0.04 & -1.96 \\
\hline V-type proton ATPase catalytic subunit A & 0.52 & 0.10 & -1.92 \\
\hline V-type proton ATPase subunit E 1 & 0.53 & 0.09 & -1.89 \\
\hline Annexin A5 & 0.70 & 0.06 & -1.43 \\
\hline Hemoglobin subunit alpha & 2.67 & 0.04 & 2.67 \\
\hline Hemoglobin subunit beta-1 & 3.60 & 0.05 & 3.60 \\
\hline
\end{tabular}

